February 2021

\title{
Ultralight Axions Versus Primordial Black Holes
}

\author{
Claudio Corianò* and Paul H. Frampton ${ }^{\dagger}$ \\ Dipartimento di Matematica e Fisica "Ennio De Giorgi", \\ Università del Salento and INFN-Lecce, \\ Via Arnesano, 73100 Lecce, Italy \\ Jihn E. Kim $\ddagger$ \\ Department of Physics, Kyung Hee University, 26 Gyungheedaero, \\ Dongdaemun-Gu, Seoul 02447, Republic of Korea
}

\begin{abstract}
We reconsider entropy arguments which have been previously argued to support the idea that the dark matter constituents are primordial black holes with many solar masses. It has recently been shown that QCD axions which solve the strong $\mathrm{CP}$ problem may have masses $m_{a}$ in the extended range $10^{-3} \mathrm{eV}>m_{a}>10^{-33} \mathrm{eV}$. Ultralight axions provide so many degrees of freedom that their entropy can exceed that of primordial black holes. This suggests that ultralight axions are more suited than primordial black holes to be constituents of dark matter.
\end{abstract}

\footnotetext{
*claudio.coriano@le.infn.it

${ }^{\dagger}$ paul.h.frampton@gmail.com

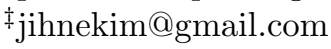




\section{Introduction}

One motivation for new physics beyond the standard model of particle theory is the strong CP problem. The absence of observed $\mathrm{CP}$ violation in strong interactions dictates that a parameter $\bar{\Theta}$ defined in terms of $\Theta$ occurring in the kinetic part of the QCD lagrangian

$$
\mathcal{L}_{Q C D}^{\text {kinetic }}=-\frac{1}{4} G_{\mu \nu}^{a} G^{\mu \nu, a}-\frac{\Theta}{64 \pi^{2}} \epsilon^{\mu \nu \alpha \beta} G_{\mu \nu}^{a} G_{\alpha \beta}^{a}
$$

by combining with a mass matrix phase

$$
\bar{\Theta}=\Theta+\arg \operatorname{det}\left(M_{u} M_{d}\right)
$$

to be invariant under chiral transformations on the quarks, satisfy

$$
\bar{\Theta}<10^{-10}
$$

This is the strong CP problem and the choice is either to accept that the fine-tuning in Eq.(3) as no more bothersome than e.g.

$$
\left|\frac{M_{\nu}}{M_{\text {top }}}\right|<10^{-12}
$$

or to adopt the orthodoxy which predicts axions [1-6] recently generalised to an ultralight axion (ULA) [7-11] with mass in an extended range

$$
10^{-33} \mathrm{eV} \leq m_{a} \leq 10^{-3} \mathrm{eV}
$$

Interestingly, this includes the mass invoked in $[12,13]$ to ameliorate problems with cold dark matter.

In the present article, we shall study ULAs as dark matter using thermodynamic arguments. 


\section{PBHs and ULAs}

Before discussing the nature of dark matter i.e. what are its constituents, we shall discuss the origin of dark matter i.e the reason why it exists. Since the first discoveries of dark matter in clusters [14] then in individual galaxies [15], a chaotic array of candidates, ranging in mass by over 100 orders of magnitude, have been proposed. To bring order from this chaos requires a guiding principle.

We shall assume that this guiding principle arises from thermodynamics. The early universe when the dark matter originated will be treated [16] as a thermodynamic system which can be dealt with using the method which was first presented by Boltzmann [17]. To be a suitable for such a treatment, there are three basic requirements:

(1) The system contains a very large number of particles.

(2) The system is close to thermal equilibrium.

(3) The system is thermodynamically isolated.

Condition (1): Boltzmann regarded Avagadro's number $N_{A} \sim 10^{24}$ as a very large number. The number of particles in the universe is $N_{U} \sim 10^{80}$, far larger than $N_{A}$, so this condition is easily satisfied.

Condition (2) appears to be satisfied because the CMB spectrum is the best black-body spectrum ever measured, strongly suggesting excellent thermal equilibrium in the early universe.

Condition (3) is the most subtle. We admit that, although this seems a very reasonable assumption, it is debatable. The reader could ask about the possibility that the universe interacts, or is entangled, with other universes. Could that not allow energy to flow in to, and out of, the universe? Indeed it could, so we exclude such scenarios.

Provided that the early universe can be treated as a thermodynamic system close to thermal equilibrium, it follows both that the entropy of the universe is a sensible concept and that because of Boltzmann's second law the entropy will only remain constant or increase. This suggests that we choose as our

\section{Guiding principle}

Subject to dynamical constraints, the dark matter constituents are such that the entropy of the universe reaches its maximum possible value. 
A brief note about notation. To abbreviate the exponents in very large numbers it will be useful to employ the symbol $\mathcal{G} \equiv 10^{100}$, For the ULAs our mass unit is the electron volt, $\mathrm{eV}$. For PBHs our mass unit is the Solar mass, $M_{\odot}$. They are related by $M_{\odot} \sim 10^{66} \mathrm{eV}$. In our discussion, we shall sometimes ignore coefficients $O(1)$ and equations will use $\sim$, not $=$. Conclusions derived therefrom about physics will, nevertheless, be ironclad.

Let $S_{U}$ be the total dimensionless entropy, that is the Boltzmann entropy divided by Boltzmann's constant, of the universe, excluding it surface.

The surface entropy equals its area in Planck units. For radius $R\left(t_{0}\right) \sim$ 44 Gly, the surface entropy is $\sim 10^{23} \mathcal{G}$. According to the holographic principle of 't Hooft [18], the highest possible entropy of the universe, whatever its content, is the entropy of its boundary surface. This provides the maximum $S_{U}\left(t_{0}\right) \leq 10^{23} \mathcal{G}$. This inequality is saturated, again according to 't Hooft [18], only if the universe itself is a black hole which is not possible because the radius $R\left(t_{0}\right) \sim 44$ Gly exceeds its Schwarzschild radius $R_{S c h}\left(t_{0}\right) \sim 30$ Gly by roughly a factor $\sim \sqrt{2}$, to be used later. Thus $S_{U}\left(t_{0}\right)<10^{23} \mathcal{G}$. A perspicuous discussion of entropy is in [19].

Let us consider a toy model for the visible universe, sufficiently realistic for the present purpose. In this model there are exactly $10^{11}$ galaxies, each with mass exactly $10^{12} M_{\odot}$.

The present universe has, excluding the dark matter, a number of components including the $\mathrm{CMB}$, relic neutrinos, stars and black holes. According to e.g. Weinberg's textbook [20], the first three contribute to $S_{U}\left(t_{0}\right)$ by the amounts $\sim 10^{-12} \mathcal{G}, \sim 10^{-12} \mathcal{G}, \sim 10^{-20} \mathcal{G}$ respectively.

It is believed that each galaxy contains near to its center a supermassive black hole (SMBH). In our toy model, we shall assume that all $10^{11}$ of these SMBHs have mass exactly $10^{7} M_{\odot}$. The Bekenstein-Hawking entropy $[21,22]$ of a black hole with mass $\eta M_{\odot}$ is $S_{B H}\left(\eta M_{\odot}\right) \sim 10^{78} \eta^{2}$. This leads to a contribution to $S_{U}\left(t_{0}\right)$ of $\sim 10^{(78+14+11)}=10^{3} \mathcal{G}$, over a trillion times more than the contributions from $\mathrm{CMB}$, relic neutrinos and stars, all added together.

We assume the dark energy does not contribute to $S_{U}$, as is the case if it is described by a cosmological constant with no degrees of freedom. All observations of dark energy are currently consistent with this interpretation.

The above discussion implies that without dark matter $S_{U}\left(t_{0}\right)$ should lie between the bounds

$$
10^{3} \mathcal{G} \leq S_{U}\left(t_{0}\right)<10^{23} \mathcal{G}
$$


Our guiding principle ut supra requires that, when dark matter is added, the value of $S_{U}\left(t_{0}\right)$ will be as near to the upper limit in Eq.(6) as possible. We believe this is what determines the choice by Nature for what is the dark matter candidate. How close to the 't Hooft holographic limit can the dark matter take us?

To answer this question, which is central to the present Letter, we revisit two facts about the visible universe that its radius $R\left(t_{0}\right) \sim 44 G l y$ while its Schwarzschild radius $R_{S c h}\left(t_{0}\right) \sim 30 G l y$. We propose that the dark matter provides enough entropy to saturate the 't Hooft bound as if the universe were a black hole! We thus use $R\left(t_{0}\right) \sim 30$ Gly and the holographic bound is reduces by a number $\sim 2$ which we shall absorb within the order of magntude. Therefore, we require that, if possible, the dark matter provide a contribution $\sim 10^{23} \mathcal{G}$.

In subsections (2.1) and (2.2), we shall study whether such a large contribution as $\sim 10^{23} \mathcal{G}$ to $S_{U}\left(t_{0}\right)$ is possible for, respectively, primordial black holes and ultralight axions.

\subsection{Primordial black holes}

It was hypothesised over five years ago [23] (see also [24-28]) that the best candidate for dark matter at that time might be intermediate mass black holes, summarised by the acronym $D M=P I M B H s$. One motivation came from the lack of any convincing particle theory candidate. A second motivation came from the fact that $D M=P I M B H s$ requires no new physics beyond the standard model of particle theory plus general relativity. Note that the PIMBHs must be primordial because there is insufficient baryonic matter to accommodate all of the dark matter. A third motivation came from entropy considerations, discussed ut infra. At that time, axions which solve the strong CP problem of QCD were believed to have mass $m_{a} \geq 10^{-12} \mathrm{eV}$. A recent extension of this lower limit on $m_{a}$, see subsection 2.2 ut infra, was the inspiration for our reconsideration of the entropy of the universe.

The paper [23] was published more than four months before the announcemnent of the discovery by LIGO [29] of gravitational waves. That dramatic discovery revealed that black holes exist with tens of $M_{\odot}$ 's, although they are probably not primordial, rather the result of gravitational collapse followed by mergers. Initially, we were interested only in dark matter within the Milky Way, since this was the most accessible to detection by microlensing [30].

Within the Milky Way, stability of the galactic disk [31] puts an upper limit on the allowed mass of PIMBHs. Although the Milky Way has an SMBH 
at its centre, $\operatorname{Sgr} A^{*}$, with mass $M \sim 4 \times 10^{6} M_{\odot}$, it is impossible that there exists a second such object in the spiral arms because, as shown in [31] by numerical simulation, this would have destabilised the galactic disk within its age. To stay away from such a disaster, in [23] we discussed the range of masses

$$
10^{2} M_{\odot} \leq M_{P I M B H} \leq 10^{5} M_{\odot}
$$

where the upper limit was inspired by [31] .

The lower limit in Eq. (7) corresponds to that in Eq. (6), ut supra, as follows. Let us suppose the dark matter in our toy model of the universe is entirely made up of black holes each with mass $10^{p} M_{\odot}$. Using the total dark matter mass $10^{23} M_{\odot}$ and the Bekenstein-Hawking formula then gives a contribution to $S_{U}\left(t_{0}\right)$ which is

$$
S_{U}\left(t_{0}\right) \sim 10^{(23-p+78+2 p)}=10^{(1+p)} \mathcal{G}
$$

so that for $p=2$ this contribution coincides with the lower bound for the entropy of the universe in Eq.(6). As we increase $p=2$ to $p=5$ as suggested in Eq.(7) the entropy of the universe increases by 3 orders of magnitude (OoMs).

In [23], we were satisfied to increase $D M=P I M B H s$ by just 3 OoMs. But the 't Hooft holographic principle, Eq.(6) suggests that the increase could be far larger, by $\sim 20$ OoMs. Is this possible by dark matter constituents which are PBHs? The upper limit in Eq.(7) applies only inside the Milky Way. Outside, the disk stability constraint is inapplicable and heavier black holes are permitted. Of course, we know about the SMBHs, one per galaxy, which were used to obtain the lower bound in Eq.(6). But what if all the dark matter were made from additional black holes of mass $\sim 10^{p} M_{\odot}$ with $p>5$ ?

We know there is dark matter associated with clusters, and it is unknown whether there are stupendously large black holes (SLABs) [32] unassociated with either galaxies or clusters. The highest $p$-value considered in [32] is $p=18$, a black hole with one hundred thousandth of the total mass of the universe. However unlikely, and contrary to the need for dark matter within galaxies, let us assume that there is a monochromatic spectrum at $p=18$, in order to maximise the entropy of the universe. From Eq.(8) we find $S_{U}\left(t_{0}\right) \sim 10^{19} \mathcal{G}$ which, in Eq.(6), succeeds in acquiring only 16 of the 20 desired OoMs. Thus, saturation of the 't Hooft holographic upper limit seems impossible, using only $\mathrm{PBH}$.

In the next subsection, by considering ultralight axions, we shall find a significantly improved method to saturate 't Hooft's upper limit. 


\subsection{Ultralight axions}

In the Introduction, we briefly discussed the QCD axion. In the canonical case both the axion mass $m_{a}^{Q C D}$ and its coupling to normal matter are inversely proportional to the the axion decay constant, $f_{a}$

$$
m_{a}^{Q C D}=\frac{\sqrt{\chi_{Q C D}}}{f_{a}} \simeq m_{\pi} f_{\pi} \frac{\sqrt{m_{u} m_{d}}}{m_{u}+m_{d}}\left(\frac{1}{f_{a}}\right)
$$

where $\chi_{Q C D}$ is the QCD topological susceptibility; $m_{u}, m_{d}$ and $m_{\pi}$ are the masses of up-quark, down-quark, and $\pi$-meson respectively.

The relations in Eq.(9) are inevitable when there is only one confining QCD gauge group. The invisible axion mass is then within the range between $1 \mathrm{peV}$ and $1 \mathrm{meV}$. Rather, we are interested in an ULA which solves the strong CP problem with suppressed $m_{a}$, if possible down to $\sim 10^{-33} \mathrm{eV}$, the minimum mass for which the axion Compton wavelength is smaller than the Hubble horizon.

One way to accomplish this [7-10] is to assume that Nature is endowed with a discrete $Z_{\mathcal{N}}$ symmetry and $\mathcal{N}$ discrete worlds linked by the axion field. As we shall discuss, this leads to a suppression of the canonical axion mass in Eq.(9) by a dependence for asymptotically large $\mathcal{N}$,

$$
m_{a} \propto m_{a}^{Q C D}\left(\frac{m_{u}}{m_{d}}\right)^{\frac{\mathcal{N}}{2}}
$$

Although Eq.(10) is strictly valid only for large $\mathcal{N}$, it is a good approximation already at low finite $\mathcal{N}$ which must be an odd integer to solve the strong $\mathrm{CP}$ problem. To suppress from the visible axion at $100 \mathrm{keV}$ to the desired mass $10^{-33} \mathrm{eV}$, and given that $z \equiv m_{u} / m_{d} \simeq 0.48$ we can estimate from Eq.(10) that $\mathcal{N}=118.75 \rightarrow 119$, the nearest odd integer. Such an $\mathcal{N}=O(100)$ theory contains an $m_{a} \sim 10^{-33} \mathrm{eV}$ axion which solves the strong $\mathrm{CP}$ problem.

Before studying the ULA contribution to the entropy of the universe, and to make our Letter self-contained, we discuss a few details of the $Z_{\mathcal{N}}$ theory to explicate two of our non-obvious assertions ut supra. It easiest to begin from $\mathcal{N}=2$ where there is only one degenerate mirror world with the $Z_{2}$ symmetry between the two standard models SM and SM'

$$
S M \leftrightarrow S M^{\prime} \quad a \rightarrow a+\pi f_{a}
$$

with the lagrangian

$$
\mathcal{L}=\mathcal{L}_{S M}+\mathcal{L}_{S M^{\prime}}+\frac{\alpha_{s}}{8 \pi}\left(\frac{a}{f_{a}}-\theta\right) G_{\mu \nu} \tilde{G}^{\mu \nu}+\frac{\alpha_{s}}{8 \pi}\left(\frac{a}{f_{a}}-\theta+\pi\right) G_{\mu \nu}^{\prime} \tilde{G}^{\prime \mu \nu}+\ldots
$$


where the ellipsis denotes $Z_{\mathcal{N}}$-symmetric portal couplings which may connect the different mirror sectors. The axion potential is more shallow than for $\mathcal{N}=1$ because of partial cancellation between terms for SM and SM', such as

$$
\left[\sqrt{m_{u}^{2}+m_{d}^{2}+2 m_{u} m_{d} \cos \left(\frac{a}{f_{a}}\right)}+\sqrt{m_{u}^{2}+m_{d}^{2}-2 m_{u} m_{d} \cos \left(\frac{a}{f_{a}}\right)}\right]
$$

In this case $\frac{a}{f_{a}}=0$ is an unstable maximum so strong CP is not solved. The origin alternates between an unstable maximum and a stable minimum for $\mathcal{N}=1,2,3, \ldots$, thus explaining the first of our two non-obvious assertions, that $\mathcal{N}$ must be an odd integer to solve the strong $\mathrm{CP}$ problem.

To understand the exponential suppression of the axion mass for large $\mathcal{N}$ of the form $m_{a} \sim z^{\frac{\mathcal{N}}{2}}$ requires a discussion of general $\mathcal{N}$. With $\mathcal{N}$ copies of the SM the $Z_{\mathcal{N}}$ symmetry is

$$
S M_{k} \rightarrow S M_{k+1} \quad a \rightarrow \frac{2 \pi k}{\mathcal{N}} f_{a} \quad 0 \leq k \leq(\mathcal{N}-1)
$$

and the lagrangian is

$$
\mathcal{L}=\Sigma_{k=0}^{\mathcal{N}-1}\left[\mathcal{L}_{S M_{k}}+\frac{\alpha_{s}}{8 \pi}\left(\theta_{a}+\frac{2 \pi k}{\mathcal{N}}\right) G_{k} \tilde{G}_{k}\right]+\ldots
$$

The resulting axion potential is

$$
V_{\mathcal{N}}\left(\theta_{a}\right)=-m_{\pi}^{2} f_{\pi}^{2} \Sigma_{k=0}^{\mathcal{N}-1} \sqrt{1-\beta \sin ^{2}\left(\frac{\theta_{a}}{2}+\frac{\pi k}{\mathcal{N}}\right)}
$$

where $\beta=4 m_{u} m_{d}\left(m_{u}+m_{d}\right)^{-2}=4 z(1+z)^{-2}$ with $z=m_{u} / m_{d}$.

In the limit $\mathcal{N} \rightarrow \infty$, the leading term is

$$
m_{a}^{2} f_{a}^{2} \sim \frac{m_{\pi}^{2} f_{\pi}^{2}}{\sqrt{\pi}} \sqrt{\frac{1-z}{1+z}} \mathcal{N}^{\frac{3}{2}} z^{\mathcal{N}} \propto\left(\frac{m_{u}}{m_{d}}\right)^{\mathcal{N}}
$$

so that the ULA mass has the suppression $m_{z} \propto z^{\frac{\mathcal{N}}{2}}$ which was quoted without justification, ut supra. The asymptotic form in Eq.(17) remains quite accurate even at the lowest nontrivial odd integer $\mathcal{N}=3$. In the large $\mathcal{N}$ limit of Eq.(17), the ULA becomes massless as the Nambu-Goldstone boson of breaking a continuous $U(1) \supset Z_{\infty}$.

In the above, we showed how the QCD axion works for this specific ULA case. For the purpose of model building, however, without introducing $\mathcal{N}$ copies, 
the same result can be obtained when we introduce a discrete symmetry $Z_{\mathcal{N}}$ into the potential.

Given an ultralight axion with mass $10^{-q} \mathrm{eV}$, solving the strong CP problem, we can estimate its contribution to the entropy of the present universe by noting that the present mass of the universe is $M_{U} \sim 10^{23} M_{\odot} \sim 10^{89} \mathrm{eV}$. If the ULA comprises all the dark matter, it contributes

$$
S_{U}\left(t_{0}\right) \sim 10^{q-11} \mathcal{G}
$$

so that for $q=33$ we come within one order of magnitude of saturating the 't Hooft holographic bound in Eq.(6). In the previous subsection, we found this is not possible for PBHs. Based on our guiding principle, we must conclude that ULAs are better suited to be the dark matters constituents than PBHs.

From the point of view of entropy alone, any ultralight scalar or pseudoscalar can as well fulfil the 't Hooft holographic bound as can an ULA.

\section{Discussion of guiding principle}

In Section 2, we enunciated our guiding principle as:" Subject to dynamical constraints, the dark matter constituents are such that the entropy of the universe reaches its maximum possible value". This is remarkably similar to the statement of the second law over 150 years ago by Clausius, the physicist who first coined the word entropy: "The entropy of the universe tends to a maximum" (1865). It is interesting that he talked about the "universe" despite the fact that his experience was limited to laboratory systems such as a box of ideal gas. Nevertheless, by applying our guiding principle to dark matter in the real universe, we have succeeded to bring order out of the chaos generated by very many candidates ranging in mass by over 117 OoMs, by reaching the more orderly selection that just two candidates, PBHs and ULAs, are consistent.

A key ingredient in our analysis has been the insight by 't Hooft [18] that the number of degrees of freedom (dofs) available for gravity in $3+1$ spacetime dimensions is not greater than the number of dofs for quantum field theory in $2+1$ spacetime dimensions. His 1993 paper on gravity was seminal to the holographic principle and to this Letter.

For PBHs of mass $10^{p} M_{\odot}$ and ULAs of mass $10^{-q} \mathrm{eV}$ respectively, taken as constituting all dark matter, our results for the present entropy of the universe are

$$
S_{U}\left(t_{0}\right) \sim 10^{1+p} \mathcal{G} \text { and } S_{U}\left(t_{0}\right) \sim 10^{q-11} \mathcal{G}
$$


In the former, even such a gigantic SLAB as $p=18$ falls a few OoMs short; in the latter, an ultralight axion at the Hubble scale $q=33$ can fully saturate the holographic bound, and it is therefore better suited as the constituent of dark matter.

That being said, $D M=P I M B H S$ [23] remains a real possibility and merits further study using microlensing of stars in the Magellanic Clouds [30]. Such an experiment employing DECam data was recently attempted at LLNL but stymied [33] by crowded field issues. A further opportunity to microlens may occur at the Rubin Observatory [34].

\section{Acknowlegements}

CC is partially supported by INFN of Italy under the QFT-HEP initiative. JEK is supported in part by the National Research Foundation grant NRF2018R1A2A3074631.

\section{References}

[1] S. Weinberg, A New Light Boson?

Phys. Rev. Lett. 40, 223 (1978).

[2] F. Wilczek, Problem of Strong $P$ and $T$ Invariance in the Presence of Instantons. Phys. Rev. Lett. 40, 279 (1978).

[3] J. E. Kim, Weak Interaction Singlet and Strong CP Invariance. Phys. Rev. Lett. 43, 103 (1979).

[4] M. A. Shifman, A. I. Vainshtein, and V. I. Zakharov, Can Confinement Ensure Natural CP Invariance of Strong Interactions? Nucl. Phys. B166, 493 (1980).

[5] A. R. Zhitnitsky, On Possible Suppression of the Axion Hadron Interactions. Sov. J. Nucl. Phys. 31, 260 (1980). [Yad. Fiz. 31, 497 (1980)].

[6] M. Dine, W. Fischler, and M. Srednicki, A Simple Solution to the Strong CP Problem with a Harmless Axion. Phys. Lett. B104, 199 (1981). 
[7] A. Hook, Solving the Hierarchy Problem Discretely.

Phys. Rev. Lett. 120, 261802 (2018). arXiv:1802.10093 [hep-ph].

[8] S. Das and A. Hook, Non-linearly Realised Discrete Symmetries. JHEP 10:071 (2020). arXiiv: 2006 .10767 [hep-ph] .

[9] L. Di Luzio, B. Gavela, P. Quilez and A. Ringwald, An even lighter $Q C D$ axion. arXiv:2102.00012 [hep-ph].

[10] L. Di Luzio, B. Gavela, P. Quilez and A. Ringwald, Dark matter from an even lighter QCD axion: trapped misalignment. arXiv:2102.01082 [hep-ph].

[11] J.E. Kim, Anomalies and parities for quintessential and ultra-light axions. arXiv:2102.01795 [hep-ph]

[12] J. E. Kim and D. J. E. Marsh, An ultralight pseudoscalar boson. Phys. Rev. D93, 0250272016. arXiv:1510.01701 [hep-ph].

[13] L. Hui, J.P. Ostriker, S. Tremaine and E. Witten, Ultralight scalars as cosmological dark matter. Phys.Rev. D 95, 043541 (2017). arXiv:1610.08297 [astro-ph.CO].

[14] F. Zwicky, Die Rotverschiebung von extragalaktischen Nebein. Helv. Phys. Acta 6, 110 (1933).

[15] V.C. Rubin, N. Thonnard and W.K. Ford, Rotational Properties of 21 SC Galaxies with a Large Range of Luminosities and Radii, from NGC4605 (R=4kpc) to UGC2885 (R=122kpc). Astrophys. J. 238, 471 (1980).

[16] P.H. Frampton, On the Origin and Nature of Dark Matter.. Int.J.Mod.Phys. A33, 1830030 (2018). arXiv:1804.03516 [physics.gen-ph]. 
[17] L.Boltzmann, Weitere Studien uber das Warmegleichgewicht unter Gasmolekulen. Sitzungsberichte der Akademie der Wissenschaften. 66, 275 (1872)

[18] G. 't Hooft, Dimensional Reduction in Quantum Gravity. in Salamfestchrift, World Scientific Publishing Company (1993).

[19] L. Susskind, The Black Hole War: My Battle with Stephen Hawking to Make the World Safe for Quantum Mechanics.

Little Brown and Company (2008).

[20] S. Weinberg,

Gravitation and Cosmology : Principles and Applications of the General Theory of Relativity.

John Wiley \& Sons. (1972).

[21] J.D. Bekenstein, Black Holes and Entropy.

Phys. Rev. D7, 2333 (1973).

[22] S.W. Hawking, Particle Creation by Black Holes. Commun. Math. Phys. 43, 199 (1975).

[23] P.H. Frampton, Searching for Dark Matter Constituents with Many Solar Masses. Mod. Phys. Lett. A31, 1650093 (2016). arXiv: 1510.00400 [hep-ph].

[24] G.F. Chapline, Cosmological Effects of Primordial Black Holes.

Nature 253, 251 (1975).

[25] G.F. Chapline, Hadron Physics and Primordial Black Holes.

Phys. Rev. D12, 2949 (1975)'

[26] G.F. Chapline and J. Barbieri, MACHO Messages from the Big Bang. LHEP 01, 17 (2018). arXiv: 1801.07345 [gr-qc] . 
[27] P.H. Frampton, M. Kawasaki, F. Takahashi and T. Yanagida, Primordial Black Holes as All Dark Matter.

JCAP 04:023 (2010).

arXiv: 1001 . 2308 [hep-ph] .

[28] G.F. Chapline and P.H. Frampton, A New Direction for Dark Matter Research:

Intermediate Mass Compact Halo Objects.

JCAP 11:042 (2016).

arXiv: 1608.04297 [gr-qc]

[29] B.P. Abbott, et al,, (LIGO Scientific and Virgo Collaborations),

Observation of Gravitational Waves from a Binary Black Hole Merger.

Phys.Rev.Lett. 116, 061102 (2016).

arXiv: 1602.03837 [gr-qc].

[30] C. Alcock, et al., (MACHO Collaboration),

The MACHO project: Microlensing results from 5.7 years

of $L M C$ observations.

Astrophys.J. 542, 281 (2000).

arXiv:astro-ph/0001272 [astro-ph].

[31] G.H. Xu and J.P. Ostriker.

Dynamics of massive black holes as a possible candidate

of Galactic dark matter.

Astrophys.J. 437, 184 (1994).

[32] B. Carr, F. Kuhnel and L. Visinelli, Constraints on Stupendously Large Black Holes.

MNRAS. 5012029 (2021)

arXiv:2008.08077 [astro-ph.CO].

[33] G.F. Chapline, private communication (February 2021)

[34] https://www.lsst.org 